\title{
On transcendental meromorphic solutions of certain type of nonlinear algebraic differential equations
}

Jianjun Zhang*

"Correspondence:
zhangjianjun1982@163.com
Mathematics and Information
Technology School, Jiangsu Second
Normal University, Nanjing, 210013,
China

\begin{abstract}
The main purpose of this paper is to give the forms of transcendental meromorphic solutions of nonlinear differential equation of the form

$$
f^{n} f^{\prime}+R(z)=p_{1}(z) e^{\alpha_{1}(z)}+p_{2}(z) e^{\alpha_{2}(z)}
$$

where $R(z)$ is a rational function, $p_{1}, p_{2}$ are nonzero rational functions and $\alpha_{1}, \alpha_{2}$ are nonconstant polynomials. More precisely, we have shown the conditions concerning $\frac{\alpha_{1}^{\prime}}{\alpha_{2}^{\prime}}$ that will ensure the existence of the possible meromorphic solutions of the above equation.
\end{abstract}

MSC: Primary 34A34; secondary 30D35

Keywords: algebraic differential equations; meromorphic solutions; growth order; poles

\section{Introduction and main results}

Let $f(z)$ be a meromorphic function in the complex plane $\mathbb{C}$. We assume that the reader is familiar with the value distribution theory of meromorphic function (see $[1,2])$ and its associated standard notations, such as $T(r, f), m(r, f), N(r, f)$ etc.

It is interesting and difficult to find the transcendental meromorphic solution of nonlinear algebraic differential equations. In the last ten years, people have shown great interest in the equation of the following form:

$$
f^{n}+Q_{d}(z, f)=p_{1}(z) e^{\alpha_{1}(z)}+p_{2}(z) e^{\alpha_{2}(z)}
$$

where $Q_{d}(z, f)$ denotes a polynomial in $f$ and its derivatives with a total degree $d \leq n-1$, with small functions of $f$ as the coefficients, $p_{1}(z), p_{2}(z)$ are rational functions and $\alpha_{1}(z)$, $\alpha_{2}(z)$ are polynomials.

In 2006, Li and Yang [3] investigated the existence of entire solution of equation (1). In 2011, Li [4] gave the forms of meromorphic solutions of equation (1) for specific $\alpha_{1}(z)$ and $\alpha_{2}(z)$. In 2013, Liao, Yang and Zhang [5] investigated the exact meromorphic solutions of equation (1), when $p_{1}(z), p_{2}(z)$ are rational functions and $\alpha_{1}(z), \alpha_{2}(z)$ are polynomials.

(c) The Author(s) 2016. This article is distributed under the terms of the Creative Commons Attribution 4.0 International License (http://creativecommons.org/licenses/by/4.0/), which permits unrestricted use, distribution, and reproduction in any medium, provided you give appropriate credit to the original author(s) and the source, provide a link to the Creative Commons license, and indicate if changes were made. 
Recently, Liao and Ye [6] got the following result when the term $f^{n}$ is replaced by $f^{n} f^{\prime}$ and the two terms on the right side of the equation become only one term in equation (1).

Theorem A Let $Q_{d}(z, f)$ be a differential polynomial in $f$ of degree d with rational function coefficients. Suppose that $u$ is a nonzero rational function and $v$ is a nonconstant polynomial. If $d \leq n-1$ and the differential equation:

$$
f^{n} f^{\prime}+Q_{d}(z, f)=u(z) e^{\nu(z)}
$$

admits a meromorphic solution $f$ with finitely many poles, then $f$ has the following form:

$$
f(z)=s(z) e^{\frac{v(z)}{n+1}} \quad \text { and } \quad Q_{d}(z, f) \equiv 0
$$

where $s(z)$ is a rational function with $s^{n}\left((n+1) s^{\prime}+v^{\prime} s\right)=(n+1) u$.

It is difficult to give the form of meromorphic solutions of the following differential equations:

$$
f^{n} f^{\prime}+Q_{d}(z, f)=p_{1}(z) e^{\alpha_{1}(z)}+p_{2}(z) e^{\alpha_{2}(z)},
$$

where $Q_{d}(z, f)$ is a differential polynomial in $f$ with small functions of $f$ as the coefficients, $p_{1}, p_{2}$ are small functions of $f, \alpha_{1}(z), \alpha_{2}(z)$ are nonconstant polynomials.

Next, we will give the possible forms of meromorphic solutions of equation (2) when $Q_{d}(z, f), p_{1}, p_{2}$ are rational functions and $\alpha_{1}(z), \alpha_{2}(z)$ are nonconstant polynomials. Now, we give our results as follows.

Theorem Let $n \geq 2$ be an integer and $R(z)$ be a rational function. Suppose that $p_{1}, p_{2}$ are nonzero rational functions and $\alpha_{1}, \alpha_{2}$ are nonconstant polynomials. If the differential equation

$$
f^{n} f^{\prime}+R(z)=p_{1}(z) e^{\alpha_{1}(z)}+p_{2}(z) e^{\alpha_{2}(z)}
$$

admits a transcendental meromorphic solution $f$, then $\frac{\alpha_{1}^{\prime}}{\alpha_{2}^{\prime}}=1, R(z) \equiv 0$, and $f(z)$ has the following form:

$$
f(z)=q(z) e^{\frac{\alpha_{1}(z)}{n+1}}
$$

where $q(z)$ is a rational function with $q^{n}\left((n+1) q^{\prime}+q \alpha_{1}^{\prime}\right)=(n+1)\left(p_{1}+p_{2} e^{\alpha_{2}(z)-\alpha_{1}(z)}\right)$.

From the theorem, we can easily obtain the following result.

Corollary Let $n \geq 2$ be an integer and $R(z)$ be a rational function. Suppose that $p_{1}, p_{2}$ are nonzero rational functions and $\alpha_{1}, \alpha_{2}$ are nonconstant polynomials. If the differential equation

$$
f^{n} f^{\prime}+R(z)=p_{1}(z) e^{\alpha_{1} z}+p_{2}(z) e^{\alpha_{2} z}
$$


admits a transcendental meromorphic solution $f$, then $\frac{\alpha_{1}}{\alpha_{2}}=1, R(z) \equiv 0$, and $f(z)$ has the following form:

$$
f(z)=q(z) e^{\frac{\alpha_{1} z}{n+1}}
$$

where $q(z)$ is a rational function with $q^{n}\left((n+1) q^{\prime}+q \alpha_{1}\right)=(n+1)\left(p_{1}+p_{2}\right)$.

Remark 1 If $R(z)$ replaced by $R(z, f)$ in the theorem, where $R(z, f)$ is a differential polynomial in $f$ with rational function as its coefficients, then the conclusion is not true generally. For example, $f(z)=e^{z}+e^{-z}$ satisfying the following differential equation:

$$
f^{2} f^{\prime}-f^{\prime}=e^{3 z}-e^{-3 z}
$$

however, $\frac{\alpha_{1}^{\prime}}{\alpha_{2}^{\prime}}=-1$ and the equation admits a solution which is not of the form $q(z) e^{P(z)}$, where $q(z)$ is a rational function and $P(z)$ is a nonconstant polynomial.

Remark 2 If $f^{n} f^{\prime}$ replaced by $f^{n}$ in the theorem, then the conclusion is not true. For example, $f(z)=e^{z}-z e^{-z}$ is a meromorphic solution of the following differential equation:

$$
f^{2}+2 z=e^{2 z}-z^{2} e^{-2 z}
$$

we have $\frac{\alpha_{1}^{\prime}}{\alpha_{2}^{\prime}}=-1$ and the equation admits a solution which is not of the form $q(z) e^{P(z)}$, where $q(z)$ is a rational function and $P(z)$ is a nonconstant polynomial.

Remark 3 If $n=1$ in the theorem, then the conclusion is not true. For example, $f(z)=$ $e^{z}+e^{-z}$ satisfies the following differential equation:

$$
f^{\prime}=e^{2 z}-e^{-2 z}
$$

Obviously, we have $\frac{\alpha_{1}^{\prime}}{\alpha_{2}^{\prime}}=-1 \neq 1$, but the equation still admits a transcendental meromorphic solution which is not of the form $q(z) e^{P(z)}$, where $q(z)$ is a rational function and $P(z)$ is a nonconstant polynomial.

In this paper, we define the degree of a rational function $R(z)=\frac{P(z)}{Q(z)} \not \equiv 0$ at $\infty$ by $\operatorname{deg}_{\infty} R=$ $\operatorname{deg} P-\operatorname{deg} Q$, where $P(z), Q(z)$ are co-prime polynomials.

\section{Some lemmas}

Lemma 1 ([7]) Let $a_{j}(z)$ be an entire function of finite order $\leq \rho$. Let $g_{j}(z)$ be entire and $g_{k}(z)-g_{j}(z)(j \neq k)$ be a transcendental entire function or polynomial of degree greater than $\rho$. Then

$$
\sum_{j=1}^{n} a_{j}(z) e^{g_{j}(z)}=a_{0}(z)
$$

holds only when

$$
a_{0}(z)=a_{1}(z)=\cdots=a_{n}(z) \equiv 0 .
$$


Lemma 2 Let $n \geq 1$ be an integer. Suppose that $R(z), p_{1}(z), p_{2}(z)$ are small functions of $f$ and $\alpha_{1}, \alpha_{2}$ are nonconstant polynomials. Iff is a meromorphic solution of the equation

$$
f^{n} f^{\prime}+R(z)=p_{1}(z) e^{\alpha_{1}(z)}+p_{2}(z) e^{\alpha_{2}(z)}
$$

then $f$ is of finite order.

Proof Obviously, any meromorphic function satisfying equation (4) must be transcendental. Denote $k=\max \left\{\operatorname{deg} \alpha_{1}, \operatorname{deg} \alpha_{2}\right\}$, by using the Clunie reasoning obtained by Yang and Ye [8], we have $m\left(r, f^{\prime}\right)=O\left(r^{k}\right)+S(r, f)$. Since every pole of $f^{\prime}$ must come from the pole of $R(z), p_{1}(z)$ or $p_{2}(z), N\left(r, f^{\prime}\right)=S(r, f)$. Therefore, we have $T\left(r, f^{\prime}\right)=O\left(r^{k}\right)+S(r, f)$ and $T\left(r, \frac{1}{f^{\prime}}\right)=O\left(r^{k}\right)+S(r, f)$. Rewriting equation (4) as follows:

$$
f^{n}=\frac{1}{f^{\prime}}\left(p_{1}(z) e^{\alpha_{1}(z)}+p_{2}(z) e^{\alpha_{2}(z)}-R(z)\right)
$$

we can obtain $T(r, f)=O\left(r^{k}\right)+S(r, f)$. Therefore, $f$ is of finite order.

\section{Proof of the theorem}

Let $f$ be a transcendental meromorphic solution of the equation (3). By Lemma 2, we know that the order of $f$ is finite. Since every pole of $f$ must come from the pole of $R(z), p_{1}(z)$ or $p_{2}(z)$, we know that $f$ has at most finitely many poles.

Next we prove that all meromorphic solutions of equation (3) must be of the form $f(z)=$ $q(z) e^{P(z)}$, where $q(z)$ is a rational function and $P(z)$ is a polynomial.

From equation (3), we have

$$
n f^{n-1}\left(f^{\prime}\right)^{2}+f^{n} f^{\prime \prime}+R^{\prime}(z)=\left(p_{1}^{\prime}+p_{1} \alpha_{1}^{\prime}\right) e^{\alpha_{1}(z)}+\left(p_{2}^{\prime}+p_{2} \alpha_{2}^{\prime}\right) e^{\alpha_{2}(z)}
$$

By eliminating $e^{\alpha_{2}(z)}$ from equations (3) and (5), we have

$$
\left(p_{2}^{\prime}+p_{2} \alpha_{2}^{\prime}\right) f^{n} f^{\prime}+\left(p_{2}^{\prime}+p_{2} \alpha_{2}^{\prime}\right) R(z)-n p_{2} f^{n-1}\left(f^{\prime}\right)^{2}-p_{2} f^{n} f^{\prime \prime}-p_{2} R^{\prime}(z)=A(z)
$$

where $A(z)=\left[p_{1}\left(p_{2}^{\prime}+p_{2} \alpha_{2}^{\prime}\right)-p_{2}\left(p_{1}^{\prime}+p_{1} \alpha_{1}^{\prime}\right)\right] e^{\alpha_{1}(z)}$.

If $A(z) \equiv 0$, then

$$
\alpha_{2}^{\prime}-\alpha_{1}^{\prime}=\frac{p_{1}^{\prime}}{p_{1}}-\frac{p_{2}^{\prime}}{p_{2}}
$$

Thus $\alpha_{2}^{\prime}-\alpha_{1}^{\prime} \equiv 0$. From equation (6), we have

$$
\left(\frac{p_{2}^{\prime}}{p_{2}}+\alpha_{2}^{\prime}\right) f^{n} f^{\prime}-n f^{n-1}\left(f^{\prime}\right)^{2}-f^{n} f^{\prime \prime}=R^{\prime}(z)-\left(\frac{p_{2}^{\prime}}{p_{2}}+\alpha_{2}^{\prime}\right) R(z) .
$$

Next, we discuss two cases.

Case 1: $R^{\prime}(z)-\left(\frac{p_{2}^{\prime}}{p_{2}}+\alpha_{2}^{\prime}\right) R(z) \equiv 0$. From equation (8), we have

$$
\left(\frac{p_{2}^{\prime}}{p_{2}}+\alpha_{2}^{\prime}\right) f^{\prime}-n\left(f^{\prime}\right)^{2}-f f^{\prime \prime}=0
$$


Suppose that $f(z)$ has infinitely many zeros, then we know from (9) that the multiplicity of each zero of $f(z)$ is not less than 2, possibly except finite many zeros of $f(z)$. Let $z_{0}$ be a zero of $f$ with multiplicity $k$, which is not a zero or pole of $\frac{p_{2}^{\prime}}{p_{2}}+\alpha_{2}^{\prime}$, then in some small neighborhood of $z_{0}$, we have $f(z)=a_{k}\left(z-z_{0}\right)^{k}+a_{k+1}\left(z-z_{0}\right)^{k+1}+\cdots$, where $a_{k}, a_{k+1}, \ldots$ are complex numbers, $a_{k} \neq 0$ and $k \geq 2$. By calculating the coefficient of the lowest power of $z-z_{0}$ in the left of equation (9), we have

$$
-n\left(k a_{k}\right)^{2}-k(k-1) a_{k}^{2}=0
$$

that is,

$$
-n k^{2}-k(k-1)=0
$$

thus $k=0$ or $k=\frac{1}{n+1}$, this is impossible. This contradiction lead to that $f$ has at most finitely many zeros. Thus, $f(z)=q(z) e^{P(z)}$, where $q(z)$ is a rational function and $P(z)$ is a polynomial.

Case 2: $R^{\prime}(z)-\left(\frac{p_{2}^{\prime}}{p_{2}}+\alpha_{2}^{\prime}\right) R(z) \not \equiv 0$. Rewriting equation (8) as follows:

$$
f^{n-1}\left(\left(\frac{p_{2}^{\prime}}{p_{2}}+\alpha_{2}^{\prime}\right) f^{\prime}-n\left(f^{\prime}\right)^{2}-f^{\prime \prime}\right)=R^{\prime}(z)-\left(\frac{p_{2}^{\prime}}{p_{2}}+\alpha_{2}^{\prime}\right) R(z)
$$

Let

$$
\left(\frac{p_{2}^{\prime}}{p_{2}}+\alpha_{2}^{\prime}\right) f^{\prime}-n\left(f^{\prime}\right)^{2}-f^{\prime \prime}=a(z)
$$

then $a(z)$ has only finitely many poles. It follows from the Clunie lemma that $m(r, a(z))=$ $O(\log r)$, thus $a(z)$ is a rational function.

If $a(z) \equiv 0$, then from equation (10), we have

$$
R^{\prime}(z)-\left(\frac{p_{2}^{\prime}}{p_{2}}+\alpha_{2}^{\prime}\right) R(z) \equiv 0
$$

this is a contradiction.

If $a(z) \not \equiv 0$, then from (10), we know that $f$ is a rational function. This is impossible.

Next we consider that $A(z) \not \equiv 0$. In order to the convenience of calculation, we denote

$$
\begin{aligned}
& A_{1}=p_{2}^{\prime}+p_{2} \alpha_{2}^{\prime}, \\
& A_{2}=p_{1}\left(p_{2}^{\prime}+p_{2} \alpha_{2}^{\prime}\right)-p_{2}\left(p_{1}^{\prime}+p_{1} \alpha_{1}^{\prime}\right) \not \equiv 0 .
\end{aligned}
$$

Thus equation (6) becomes

$$
A_{1} f^{n} f^{\prime}-n p_{2} f^{n-1}\left(f^{\prime}\right)^{2}-p_{2} f^{n} f^{\prime \prime}+R_{1}(z)=A_{2} e^{\alpha_{1}(z)},
$$


where $R_{1}(z)=\left(p_{2}^{\prime}+p_{2} \alpha_{2}^{\prime}\right) R(z)-p_{2} R^{\prime}(z)$ is a rational functions. Differentiating both sides of (12), we have

$$
\begin{aligned}
& A_{1}^{\prime} f^{n} f^{\prime}+n\left(A_{1}-p_{2}^{\prime}\right) f^{n-1}\left(f^{\prime}\right)^{2}+\left(A_{1}-p_{2}^{\prime}\right) f^{n} f^{\prime \prime}-n(n-1) p_{2} f^{n-2}\left(f^{\prime}\right)^{3} \\
& -3 n p_{2} f^{n-1} f^{\prime} f^{\prime \prime}-p_{2} f^{n} f^{\prime \prime \prime}+R_{1}^{\prime}(z)=\left(A_{2}^{\prime}+A_{2} \alpha_{1}^{\prime}(z)\right) e^{\alpha_{1}(z)} .
\end{aligned}
$$

By eliminating $e^{\alpha_{1}(z)}$ from equations (12) and (13), we obtain

$$
\begin{aligned}
& B_{1} f^{n} f^{\prime}-B_{2} f^{n-1}\left(f^{\prime}\right)^{2}-B_{3} f^{n} f^{\prime \prime}+n(n-1) p_{2} A_{2} f^{n-2}\left(f^{\prime}\right)^{3} \\
& \quad+3 n p_{2} A_{2} f^{n-1} f^{\prime} f^{\prime \prime}+p_{2} A_{2} f^{n} f^{\prime \prime \prime}=Q_{1}(z),
\end{aligned}
$$

where

$$
\begin{aligned}
& B_{1}(z)=A_{1}\left(A_{2}^{\prime}+A_{2} \alpha_{1}^{\prime}(z)\right)-A_{2} A_{1}^{\prime}, \\
& B_{2}(z)=n p_{2}\left(A_{2}^{\prime}+A_{2} \alpha_{1}^{\prime}(z)\right)+n\left(A_{1}-p_{2}^{\prime}\right) A_{2}, \\
& B_{3}(z)=p_{2}\left(A_{2}^{\prime}+A_{2} \alpha_{1}^{\prime}(z)\right)+\left(A_{1}-p_{2}^{\prime}\right) A_{2}, \\
& Q_{1}(z)=-\left(A_{2}^{\prime}+A_{2} \alpha_{1}^{\prime}(z)\right) R_{1}(z)+A_{2} R_{1}^{\prime}(z),
\end{aligned}
$$

are rational functions.

From equation (14), we have

$$
\begin{aligned}
& f^{n-2}\left(B_{1} f^{2} f^{\prime}-B_{2} f\left(f^{\prime}\right)^{2}-B_{3} f^{2} f^{\prime \prime}+n(n-1) p_{2} A_{2}\left(f^{\prime}\right)^{3}\right. \\
& \left.+3 n p_{2} A_{2} f^{\prime} f^{\prime \prime}+p_{2} A_{2} f^{2} f^{\prime \prime \prime}\right)=Q_{1}(z) .
\end{aligned}
$$

Let

$$
B_{1} f^{2} f^{\prime}-B_{2} f\left(f^{\prime}\right)^{2}-B_{3} f^{2} f^{\prime \prime}+n(n-1) p_{2} A_{2}\left(f^{\prime}\right)^{3}+3 n p_{2} A_{2} f^{\prime} f^{\prime \prime}+p_{2} A_{2} f^{2} f^{\prime \prime \prime}=Q(z)
$$

It follows from the Clunie lemma that $Q(z)$ is a rational function.

First we assume that $Q(z) \equiv 0$. Suppose that $f(z)$ has infinitely many zeros, then we know from (16) that the multiplicity of each zero of $f(z)$ is not less than 2, possibly except finite many zeros of $f(z)$. Let $z_{0}$ be a zero of $f$ with multiplicity $k$, which is not a zero or pole of $B_{1}, B_{2}, B_{3}$ and $p_{2} A_{2}$, then $f(z)=a_{k}\left(z-z_{0}\right)^{k}+a_{k+1}\left(z-z_{0}\right)^{k+1}+\cdots\left(a_{k} \neq 0, k \geq 2\right)$ holds in some small neighborhood of $z_{0}$. By calculating the coefficient of the lowest power of $z-z_{0}$ in the left of equation (16), we have

$$
n(n-1)\left(k a_{k}\right)^{3}+3 n k^{2}(k-1) a_{k}^{3}+k(k-1)(k-2) a_{k}^{3}=0,
$$

thus

$$
n(n-1) k^{2}+3 n k(k-1)+(k-1)(k-2)=0,
$$

this is impossible since $n \geq 2$ and $k \geq 2$. This contradiction lead to that $f$ has at most finitely many zeros. Thus, $f(z)=q(z) e^{P(z)}$, where $q(z)$ is a rational function and $P(z)$ is a polynomial. 
Next we assume that $Q(z) \not \equiv 0$. By differentiating (16), we have

$$
\begin{aligned}
& B_{1}^{\prime} f^{2} f^{\prime}+\left(2 B_{1}-B_{2}^{\prime}\right) f\left(f^{\prime}\right)^{2}+\left(B_{1}-B_{3}^{\prime}\right) f^{2} f^{\prime \prime}+\left(n(n-1)\left(p_{2} A_{2}\right)^{\prime}-B_{2}\right)\left(f^{\prime}\right)^{3} \\
& +\left(3 n\left(p_{2} A_{2}\right)^{\prime}-2 B_{2}-2 B_{3}\right) f^{\prime} f^{\prime \prime}+\left(\left(p_{2} A_{2}\right)^{\prime}-B_{3}\right) f^{2} f^{\prime \prime \prime}+3 n^{2} p_{2} A_{2}\left(f^{\prime}\right)^{2} f^{\prime \prime} \\
& +3 n p_{2} A_{2} f\left(f^{\prime \prime}\right)^{2}+(3 n+2) p_{2} A_{2} f^{\prime} f^{\prime \prime \prime}+p_{2} A_{2} f^{2} f^{(4)}=Q^{\prime}(z) .
\end{aligned}
$$

It follows from equations (16) and (17) that

$$
\begin{aligned}
& \left(B_{1} Q^{\prime}-B_{1}^{\prime} Q\right) f^{2} f^{\prime}-\left(B_{2} Q^{\prime}+\left(2 B_{1}-B_{2}^{\prime}\right) Q\right) f\left(f^{\prime}\right)^{2}-\left(B_{3} Q^{\prime}+\left(B_{1}-B_{3}^{\prime}\right) Q\right) f^{2} f^{\prime \prime} \\
& \quad+\left(n(n-1) p_{2} A_{2} Q^{\prime}-n(n-1)\left(p_{2} A_{2}\right)^{\prime} Q+B_{2} Q\right)\left(f^{\prime}\right)^{3} \\
& +\left(3 n p_{2} A_{2} Q^{\prime}-\left(3 n\left(p_{2} A_{2}\right)^{\prime}-2 B_{2}-2 B_{3}\right) Q\right) f^{\prime} f^{\prime \prime} \\
& \quad+\left(p_{2} A_{2} Q^{\prime}-\left(\left(p_{2} A_{2}\right)^{\prime}-B_{3}\right) Q\right) f^{2} f^{\prime \prime \prime}-3 n^{2} p_{2} A_{2} Q\left(f^{\prime}\right)^{2} f^{\prime \prime}-3 n p_{2} A_{2} Q f\left(f^{\prime \prime}\right)^{2} \\
& \quad-(3 n+2) p_{2} A_{2} Q f f^{\prime} f^{\prime \prime \prime}-p_{2} A_{2} Q f^{2} f^{(4)}=0 .
\end{aligned}
$$

If $f(z)$ has infinitely many zeros and $z_{0}$ is a zero of $f(z)$ which is not a zero or pole of the coefficients in (16) and (18), then by (16) we know that a zero $z_{0}$ of $f(z)$ is simple, and it follows from (18) that $z_{0}$ is a zero of $3 n^{2} p_{2} A_{2} Q f^{\prime \prime}-\left(n(n-1) p_{2} A_{2} Q^{\prime}-n(n-1)\left(p_{2} A_{2}\right)^{\prime} Q+\right.$ $\left.B_{2} Q\right) f^{\prime}$. Let

$$
b(z)=\frac{3 n^{2} p_{2} A_{2} Q f^{\prime \prime}-\left(n(n-1) p_{2} A_{2} Q^{\prime}-n(n-1)\left(p_{2} A_{2}\right)^{\prime} Q+B_{2} Q\right) f^{\prime}}{p_{2} A_{2} Q f} .
$$

Then $b(z)$ has only finitely many poles and it follows from the lemma of the logarithmic derivative that $m(r, b(z))=O(\log r)$. Hence $b(z)$ is a rational function.

If $b(z) \equiv 0$, then we have

$$
\begin{aligned}
\overline{f^{\prime \prime}} & =\frac{n(n-1) p_{2} A_{2} Q^{\prime}-n(n-1)\left(p_{2} A_{2}\right)^{\prime} Q+B_{2} Q}{3 n^{2} p_{2} A_{2} Q} \\
& =\frac{n-1}{3 n} \frac{Q^{\prime}}{Q}-\frac{n-1}{3 n} \frac{A_{2}^{\prime}}{A_{2}}-\frac{n-1}{3 n} \frac{p_{2}^{\prime}}{p_{2}}+\frac{1}{3 n^{2}} \frac{B_{2}}{p_{2} A_{2}} .
\end{aligned}
$$

Noticing that

$$
B_{2}(z)=n p_{2}\left(A_{2}^{\prime}+A_{2} \alpha_{1}^{\prime}\right)+n p_{2} \alpha_{2}^{\prime} A_{2}
$$

thus we have

$$
\frac{f^{\prime \prime}}{f^{\prime}}=\frac{n-1}{3 n} \frac{Q^{\prime}}{Q}-\frac{n-2}{3 n} \frac{A_{2}^{\prime}}{A_{2}}-\frac{n-1}{3 n} \frac{p_{2}^{\prime}}{p_{2}}+\frac{1}{3 n}\left(\alpha_{1}^{\prime}+\alpha_{2}^{\prime}\right) .
$$

By solving the above equation, we obtain

$$
f^{\prime}(z)=m(z) e^{\frac{\alpha_{1}+\alpha_{2}}{3 n}}
$$

where $m(z)=\left(\frac{C Q^{n-1}}{p_{2}^{n-1} A_{2}^{n-2}}\right)^{\frac{1}{3 n}}$ is a rational function. 
From equation (18) and the fact $b(z) \equiv 0$, we have

$$
\begin{aligned}
& \left(B_{1} Q^{\prime}-B_{1}^{\prime} Q\right) f^{\prime}-\left(B_{2} Q^{\prime}+\left(2 B_{1}-B_{2}^{\prime}\right) Q\right)\left(f^{\prime}\right)^{2}-\left(B_{3} Q^{\prime}+\left(B_{1}-B_{3}^{\prime}\right) Q\right) f^{\prime \prime} \\
& \quad+\left(3 n p_{2} A_{2} Q^{\prime}-\left(3 n\left(p_{2} A_{2}\right)^{\prime}-2 B_{2}-2 B_{3}\right) Q\right) f^{\prime} f^{\prime \prime} \\
& \quad+\left(p_{2} A_{2} Q^{\prime}-\left(\left(p_{2} A_{2}\right)^{\prime}-B_{3}\right) Q\right) f^{\prime \prime \prime}-3 n p_{2} A_{2} Q\left(f^{\prime \prime}\right)^{2} \\
& \quad-(3 n+2) p_{2} A_{2} Q f^{\prime} f^{\prime \prime \prime}-p_{2} A_{2} Q f^{(4)}=0 .
\end{aligned}
$$

By calculating and substituting $f^{\prime}, f^{\prime \prime}, f^{\prime \prime \prime}, f^{(4)}$ into the above equation, we can obtain

$$
s(z) f+t(z) e^{\frac{\alpha_{1}+\alpha_{2}}{3 n}}=0
$$

where

$$
\begin{aligned}
s(z)= & \left(B_{1} Q^{\prime}-B_{1}^{\prime} R\right) m(z)-\left(B_{3} Q^{\prime}+\left(B_{1}-B_{3}^{\prime}\right) Q\right)\left(m^{\prime}(z)+\frac{1}{3 n} m(z)\left(\alpha_{1}^{\prime}+\alpha_{2}^{\prime}\right)\right) \\
& +\left(p_{2} A_{2} Q^{\prime}-\left(\left(p_{2} A_{2}\right)^{\prime}-B_{3}\right) Q\right)\left(m^{\prime \prime}(z)+\frac{1}{3 n} m(z)\left(\alpha_{1}^{\prime \prime}+\alpha_{2}^{\prime \prime}\right)\right. \\
& \left.+\frac{2}{3 n} m^{\prime}(z)\left(\alpha_{1}^{\prime}+\alpha_{2}^{\prime}\right)+\frac{1}{9 n^{2}} m(z)\left(\alpha_{1}^{\prime}+\alpha_{2}^{\prime}\right)^{2}\right) \\
& -p_{2} A_{2} Q\left(m^{\prime \prime \prime}(z)+\frac{1}{n} m^{\prime}(z)\left(\alpha_{1}^{\prime \prime}+\alpha_{2}^{\prime \prime}\right)+\frac{1}{3 n} m(z)\left(\alpha_{1}^{\prime \prime \prime}+\alpha_{2}^{\prime \prime \prime}\right)+\frac{1}{n} m^{\prime \prime}(z)\left(\alpha_{1}^{\prime}+\alpha_{2}^{\prime}\right)\right. \\
& \left.+\frac{1}{3 n^{2}} m^{\prime}(z)\left(\alpha_{1}^{\prime}+\alpha_{2}^{\prime}\right)^{2}+\frac{1}{3 n^{2}} m(z)\left(\alpha_{1}^{\prime}+\alpha_{2}^{\prime}\right)\left(\alpha_{1}^{\prime \prime}+\alpha_{2}^{\prime \prime}\right)+\frac{1}{27 n^{3}} m(z)\left(\alpha_{1}^{\prime}+\alpha_{2}^{\prime}\right)^{3}\right), \\
t(z)= & -\left(B_{2} Q^{\prime}+\left(2 B_{1}-B_{2}^{\prime}\right) Q\right) m^{2}(z)-3 n p_{2} A_{2} Q\left(m^{\prime}(z)+\frac{1}{3 n} m(z)\left(\alpha_{1}^{\prime}+\alpha_{2}^{\prime}\right)\right)^{2} \\
& +\left(3 n p_{2} A_{2} Q^{\prime}-\left(3 n\left(p_{2} A_{2}\right)^{\prime}-2 B_{2}-2 B_{3}\right) Q\right) m(z)\left(m^{\prime}(z)+\frac{1}{3 n} m(z)\left(\alpha_{1}^{\prime}+\alpha_{2}^{\prime}\right)\right) \\
& -(3 n+2) p_{2} A_{2} Q m(z)\left(m^{\prime \prime}(z)+\frac{1}{3 n} m(z)\left(\alpha_{1}^{\prime \prime}+\alpha_{2}^{\prime \prime}\right)\right. \\
& \left.+\frac{2}{3 n} m^{\prime}(z)\left(\alpha_{1}^{\prime}+\alpha_{2}^{\prime}\right)+\frac{1}{9 n^{2}} m(z)\left(\alpha_{1}^{\prime}+\alpha_{2}^{\prime}\right)^{2}\right) .
\end{aligned}
$$

Next we show that $s(z) \not \equiv 0$ and $t(z) \not \equiv 0$. Suppose that the assertion is not correct. Obviously, If $s(z) \equiv 0$, then from (20), we must have $t(z) \equiv 0$. By $s(z) \equiv 0$, we have

$$
\begin{aligned}
\frac{s(z)}{m(z) p_{2} A_{2} Q}= & \frac{B_{1}}{p_{2} A_{2}} \frac{Q^{\prime}}{Q}-\frac{B_{1}^{\prime}}{p_{2} A_{2}} \\
& -\left(\frac{B_{3}}{p_{2} A_{2}} \frac{Q^{\prime}}{Q}+\frac{B_{1}}{p_{2} A_{2}}-\frac{B_{3}^{\prime}}{p_{2} A_{2}}\right)\left(\frac{m^{\prime}(z)}{m(z)}+\frac{1}{3 n}\left(\alpha_{1}^{\prime}+\alpha_{2}^{\prime}\right)\right) \\
& +\left(\frac{Q^{\prime}}{Q}-\frac{\left(p_{2} A_{2}\right)^{\prime}}{p_{2} A_{2}}+\frac{B_{3}}{p_{2} A_{2}}\right)\left(\frac{m^{\prime \prime}(z)}{m(z)}+\frac{1}{3 n}\left(\alpha_{1}^{\prime \prime}+\alpha_{2}^{\prime \prime}\right)+\frac{2}{3 n} \frac{m^{\prime}(z)}{m(z)}\left(\alpha_{1}^{\prime}+\alpha_{2}^{\prime}\right)\right. \\
& \left.+\frac{1}{9 n^{2}}\left(\alpha_{1}^{\prime}+\alpha_{2}^{\prime}\right)^{2}\right) \\
& -\left(\frac{m^{\prime \prime \prime}(z)}{m(z)}+\frac{1}{n} \frac{m^{\prime}(z)}{m(z)}\left(\alpha_{1}^{\prime \prime}+\alpha_{2}^{\prime \prime}\right)+\frac{1}{3 n}\left(\alpha_{1}^{\prime \prime \prime}+\alpha_{2}^{\prime \prime \prime}\right)+\frac{1}{n} \frac{m^{\prime \prime}(z)}{m(z)}\left(\alpha_{1}^{\prime}+\alpha_{2}^{\prime}\right)\right.
\end{aligned}
$$




$$
\begin{aligned}
& +\frac{1}{3 n^{2}} \frac{m^{\prime}(z)}{m(z)}\left(\alpha_{1}^{\prime}+\alpha_{2}^{\prime}\right)^{2}+\frac{1}{3 n^{2}}\left(\alpha_{1}^{\prime}+\alpha_{2}^{\prime}\right)\left(\alpha_{1}^{\prime \prime}+\alpha_{2}^{\prime \prime}\right) \\
& \left.+\frac{1}{27 n^{3}}\left(\alpha_{1}^{\prime}+\alpha_{2}^{\prime}\right)^{3}\right) \\
\equiv & 0
\end{aligned}
$$

Similarly, by $t(z) \equiv 0$, we can get

$$
\begin{aligned}
\frac{t(z)}{m^{2}(z) p_{2} A_{2} Q}= & -\frac{B_{2}}{p_{2} A_{2}} \frac{Q^{\prime}}{Q}-\frac{2 B_{1}}{p_{2} A_{2}}+\frac{B_{2}^{\prime}}{p_{2} A_{2}}-3 n\left(\frac{m^{\prime}(z)}{m(z)}+\frac{1}{3 n}\left(\alpha_{1}^{\prime}+\alpha_{2}^{\prime}\right)\right)^{2} \\
& +\left(3 n \frac{Q^{\prime}}{Q}-3 n \frac{\left(p_{2} A_{2}\right)^{\prime}}{p_{2} A_{2}}+\frac{2 B_{2}}{p_{2} A_{2}}+\frac{2 B_{3}}{p_{2} A_{2}}\right)\left(\frac{m^{\prime}(z)}{m(z)}+\frac{1}{3 n}\left(\alpha_{1}^{\prime}+\alpha_{2}^{\prime}\right)\right) \\
& -(3 n+2)\left(\frac{m^{\prime \prime}(z)}{m(z)}+\frac{1}{3 n}\left(\alpha_{1}^{\prime \prime}+\alpha_{2}^{\prime \prime}\right)+\frac{2}{3 n} \frac{m^{\prime}(z)}{m(z)}\left(\alpha_{1}^{\prime}+\alpha_{2}^{\prime}\right)\right. \\
& \left.+\frac{1}{9 n^{2}}\left(\alpha_{1}^{\prime}+\alpha_{2}^{\prime}\right)^{2}\right) \\
\equiv & 0,
\end{aligned}
$$

where

$$
\begin{aligned}
\frac{B_{1}}{p_{2} A_{2}} & =\left(\frac{p_{2}^{\prime}}{p_{2}}+\alpha_{2}^{\prime}\right)\left(\frac{A_{2}^{\prime}}{A_{2}}-\frac{A_{1}^{\prime}}{A_{1}}+\alpha_{1}^{\prime}\right), \\
\frac{B_{2}}{p_{2} A_{2}} & =n\left(\alpha_{1}^{\prime}+\alpha_{2}^{\prime}\right)+n \frac{A_{2}^{\prime}}{A_{2}}, \\
\frac{B_{3}}{p_{2} A_{2}} & =\alpha_{1}^{\prime}+\alpha_{2}^{\prime}+\frac{A_{2}^{\prime}}{A_{2}}, \\
\frac{B_{1}^{\prime}}{p_{2} A_{2}} & =\left(\frac{p_{2}^{\prime}}{p_{2}}+\alpha_{2}^{\prime}\right)\left(\frac{A_{2}^{\prime \prime}}{A_{2}}+\frac{A_{2}^{\prime}}{A_{2}} \alpha_{1}^{\prime}+\frac{A_{1}^{\prime}}{A_{1}} \alpha_{1}^{\prime}-\frac{A_{1}^{\prime \prime}}{A_{1}}+\alpha_{1}^{\prime \prime}\right), \\
\frac{B_{2}^{\prime}}{p_{2} A_{2}} & =n\left(\frac{p_{2}^{\prime}}{p_{2}} \frac{A_{2}^{\prime}}{A_{2}}+\frac{A_{2}^{\prime \prime}}{A_{2}}+\left(\frac{A_{2}^{\prime}}{A_{2}}+\frac{p_{2}^{\prime}}{p_{2}}\right)\left(\alpha_{1}^{\prime}+\alpha_{2}^{\prime}\right)+\alpha_{1}^{\prime \prime}+\alpha_{2}^{\prime \prime}\right), \\
\frac{B_{3}^{\prime}}{p_{2} A_{2}} & =\frac{p_{2}^{\prime}}{p_{2}} \frac{A_{2}^{\prime}}{A_{2}}+\frac{A_{2}^{\prime \prime}}{A_{2}}+\left(\frac{A_{2}^{\prime}}{A_{2}}+\frac{p_{2}^{\prime}}{p_{2}}\right)\left(\alpha_{1}^{\prime}+\alpha_{2}^{\prime}\right)+\alpha_{1}^{\prime \prime}+\alpha_{2}^{\prime \prime} .
\end{aligned}
$$

From (21) and (22), we see that the highest degree terms may appear in

$$
-\frac{1}{3 n} \alpha_{1}^{\prime} \alpha_{2}^{\prime}\left(\alpha_{1}^{\prime}+\alpha_{2}^{\prime}\right)+\frac{1}{9 n^{2}}\left(\alpha_{1}^{\prime}+\alpha_{2}^{\prime}\right)^{3}-\frac{1}{27 n^{3}}\left(\alpha_{1}^{\prime}+\alpha_{2}^{\prime}\right)^{3}
$$

and

$$
-2 \alpha_{1}^{\prime} \alpha_{2}^{\prime}-\frac{1}{3 n}\left(\alpha_{1}^{\prime}+\alpha_{2}^{\prime}\right)^{2}+\frac{2(n+1)}{3 n}\left(\alpha_{1}^{\prime}+\alpha_{2}^{\prime}\right)^{2}-\frac{3 n+2}{9 n^{2}}\left(\alpha_{1}^{\prime}+\alpha_{2}^{\prime}\right)^{2} .
$$

Namely,

$$
-\frac{1}{3 n} \alpha_{1}^{\prime} \alpha_{2}^{\prime}\left(\alpha_{1}^{\prime}+\alpha_{2}^{\prime}\right)+\frac{3 n-1}{27 n^{3}}\left(\alpha_{1}^{\prime}+\alpha_{2}^{\prime}\right)^{3}
$$


and

$$
-2 \alpha_{1}^{\prime} \alpha_{2}^{\prime}+\frac{6 n^{2}-2}{9 n^{2}}\left(\alpha_{1}^{\prime}+\alpha_{2}^{\prime}\right)^{2}
$$

Let $\alpha_{1}(z)=a_{p} z^{p}+a_{p-1} z^{p-1}+\cdots+a_{0}, \alpha_{2}(z)=b_{q} z^{q}+b_{q-1} z^{q-1}+\cdots+b_{0}$, where $a_{i}$ $(i=0,1, \ldots, p), b_{j}(i=0,1, \ldots, q)$ are complex numbers, $a_{p} b_{q} \neq 0$ and $p \geq 1, q \geq 1$.

If $p \neq q$, without loss of generality, we assume that $p>q$. Since $t(z) \equiv 0$, by (24) we have $\frac{6 n^{2}-2}{9 n^{2}} p^{2} a_{p}^{2}=0$, so $n^{2}=\frac{1}{3}$, this is impossible. Next we suppose that $p=q$.

If $a_{p}+b_{p}=0$, then by $t(z) \equiv 0$ and (24), we have $a_{p} b_{p}=0$, this is a contradiction.

If $a_{p}+b_{p} \neq 0$, then from $s(z) \equiv 0, t(z) \equiv 0,(23)$ and (24), we can obtain $-\frac{1}{3 n} a_{p} b_{p}+$ $\frac{3 n-1}{27 n^{3}}\left(a_{p}+b_{p}\right)^{2}=0$ and $-2 a_{p} b_{p}+\frac{6 n^{2}-2}{9 n^{2}}\left(a_{p}+b_{p}\right)^{2}=0$, thus we have $\frac{2(3 n-1)}{27 n^{3}}=\frac{1}{3 n} \frac{6 n^{2}-2}{9 n^{2}}$. So $n=0$ or $n=1$, this is a contradiction with the assumption $n \geq 2$.

Therefore, $s(z) \not \equiv 0$ and $t(z) \not \equiv 0$. Hence $f(z)=-\frac{t(z)}{s(z)} e^{\frac{\alpha_{1}+\alpha_{2}}{3 n}}$. Obviously, $\alpha_{1}^{\prime}(z)+\alpha_{2}^{\prime}(z) \not \equiv 0$, otherwise, $f(z)$ is a rational function, this is impossible. Thus, $f(z)$ is of the form $q(z) e^{P(z)}$, where $q(z)=-\frac{t(z)}{s(z)}$ is a rational function, $P(z)=\frac{\alpha_{1}+\alpha_{2}}{3 n}$ is a nonconstant polynomial.

Next we assume that $b(z) \not \equiv 0$. From (18) and (19), we get

$$
\begin{aligned}
& \left(B_{1} Q^{\prime}-B_{1}^{\prime} Q\right) f^{\prime}-\left(B_{2} Q^{\prime}+\left(2 B_{1}-B_{2}^{\prime}\right) Q\right)\left(f^{\prime}\right)^{2}-\left(B_{3} Q^{\prime}+\left(B_{1}-B_{3}^{\prime}\right) Q\right) f^{\prime \prime} \\
& +\left(3 n p_{2} A_{2} Q^{\prime}-\left(3 n\left(p_{2} A_{2}\right)^{\prime}-2 B_{2}-2 B_{3}\right) Q\right) f^{\prime} f^{\prime \prime} \\
& +\left(p_{2} A_{2} Q^{\prime}-\left(\left(p_{2} A_{2}\right)^{\prime}-B_{3}\right) Q\right) f^{\prime \prime \prime}-3 n p_{2} A_{2} Q\left(f^{\prime \prime}\right)^{2} \\
& \quad-(3 n+2) p_{2} A_{2} Q f^{\prime} f^{\prime \prime \prime}-p_{2} A_{2} Q f^{(4)}=b(z) p_{2} A_{2} Q\left(f^{\prime}\right)^{2}
\end{aligned}
$$

and

$$
\begin{aligned}
f^{\prime \prime} & =\frac{n(n-1) p_{2} A_{2} Q^{\prime}-n(n-1)\left(p_{2} A_{2}\right)^{\prime} Q+B_{2} Q}{3 n^{2} p_{2} A_{2} Q} f^{\prime}+\frac{p_{2} A_{2} Q b(z)}{3 n^{2} p_{2} A_{2} Q} f \\
& =\left(\frac{n-1}{3 n} \frac{Q^{\prime}}{Q}-\frac{n-1}{3 n} \frac{\left(p_{2} A_{2}\right)^{\prime}}{p_{2} A_{2}}+\frac{1}{3 n^{2}} \frac{B_{2}}{p_{2} A_{2}}\right) f^{\prime}+\frac{1}{3 n^{2}} b(z) f \\
& =\left(\frac{n-1}{3 n} \frac{Q^{\prime}}{Q}-\frac{n-1}{3 n} \frac{\left(p_{2} A_{2}\right)^{\prime}}{p_{2} A_{2}}+\frac{1}{3 n} \frac{A_{2}^{\prime}}{A_{2}}+\frac{1}{3 n}\left(\alpha_{1}^{\prime}+\alpha_{2}^{\prime}\right)\right) f^{\prime}+\frac{1}{3 n^{2}} b(z) f .
\end{aligned}
$$

Let $\varphi(z)=\frac{n-1}{3 n} \frac{Q^{\prime}}{Q}-\frac{n-1}{3 n} \frac{\left(p_{2} A_{2}\right)^{\prime}}{p_{2} A_{2}}+\frac{1}{3 n} \frac{A_{2}^{\prime}}{A_{2}}+\frac{1}{3 n}\left(\alpha_{1}^{\prime}+\alpha_{2}^{\prime}\right)$, then by calculating and substituting $f^{\prime \prime}$, $f^{\prime \prime \prime}, f^{(4)}$ into (25), we can get

$$
\mu_{1}(z) f^{2}+\mu_{2}(z) f f^{\prime}+\mu_{3}(z)\left(f^{\prime}\right)^{2}=0
$$

where

$$
\begin{aligned}
\mu_{1}(z)= & \frac{1}{3 n^{2}}\left(\varphi b+b^{\prime}\right)\left(p_{2} A_{2} Q^{\prime}-\left(\left(p_{2} A_{2}\right)^{\prime}-B_{3}\right) Q\right)-\frac{1}{3 n^{2}} b\left(B_{3} Q^{\prime}+\left(B_{1}-B_{3}^{\prime}\right) Q\right) \\
& -p_{2} A_{2} Q\left(\frac{1}{3 n^{2}} \varphi^{2} b+\frac{2}{3 n^{2}} \varphi^{\prime} b+\frac{1}{3 n^{2}} \varphi b^{\prime}+\frac{1}{3 n^{2}} b^{\prime \prime}+\frac{3 n+1}{9 n^{4}} b^{2}\right), \\
\mu_{2}(z)= & B_{1} Q^{\prime}-B_{1}^{\prime} Q-\varphi B_{3} Q^{\prime}-\varphi\left(B_{1}-B_{3}^{\prime}\right) Q+\frac{1}{n} p_{2} A_{2} Q^{\prime} b-\frac{1}{n}\left(p_{2} A_{2}\right)^{\prime} Q b \\
& +\frac{2}{3 n^{2}}\left(B_{2}+B_{3}\right) Q b+\left(p_{2} A_{2} Q^{\prime}-\left(\left(p_{2} A_{2}\right)^{\prime}-B_{3}\right) Q\right)\left(\varphi^{2}+\varphi^{\prime}+\frac{1}{3 n^{2}} b\right)
\end{aligned}
$$




$$
\begin{aligned}
& -p_{2} A_{2} Q\left(\varphi^{3}+3 \varphi \varphi^{\prime}+\varphi^{\prime \prime}+\frac{9 n+4}{3 n^{2}} \varphi b+\frac{3 n+4}{3 n^{2}} b^{\prime}\right), \\
\mu_{3}(z)= & 3 n p_{2} A_{2} Q^{\prime} \varphi-\left(3 n\left(p_{2} A_{2}\right)^{\prime}-2 B_{2}-2 B_{3}\right) Q \varphi-\left(B_{2} Q^{\prime}+\left(2 B_{1}-B_{2}^{\prime}\right) Q\right) \\
& -p_{2} A_{2} Q b-(3 n+2) p_{2} A_{2} Q\left(\varphi^{2}+\varphi^{\prime}+\frac{1}{3 n^{2}} b\right)-3 n p_{2} A_{2} R \varphi^{2},
\end{aligned}
$$

are rational functions.

We assume that $\mu_{1}(z) \equiv 0, \mu_{2}(z) \equiv 0, \mu_{3}(z) \equiv 0$ hold simultaneously. By $\mu_{1}(z) \equiv 0$, we have

$$
\begin{gathered}
\frac{1}{3 n^{2}}\left(\varphi+\frac{b^{\prime}}{b}\right)\left(\frac{Q^{\prime}}{Q}-\frac{\left(p_{2} A_{2}\right)^{\prime}}{p_{2} A_{2}}+\frac{B_{3}}{p_{2} A_{2}}\right)-\frac{1}{3 n^{2}}\left(\frac{B_{3}}{p_{2} A_{2}} \frac{Q^{\prime}}{Q}+\frac{B_{1}}{p_{2} A_{2}}-\frac{B_{3}^{\prime}}{p_{2} A_{2}}\right) \\
-\left(\frac{1}{3 n^{2}} \varphi^{2}+\frac{2}{3 n^{2}} \varphi^{\prime}+\frac{1}{3 n^{2}} \varphi \frac{b^{\prime}}{b}+\frac{1}{3 n^{2}} \frac{b^{\prime \prime}}{b}+\frac{3 n+1}{9 n^{4}} b\right) \equiv 0 .
\end{gathered}
$$

By $\mu_{2}(z) \equiv 0$, we have

$$
\begin{gathered}
\frac{B_{1}}{p_{2} A_{2}} \frac{Q^{\prime}}{Q}-\frac{B_{1}^{\prime}}{p_{2} A_{2}}-\varphi \frac{B_{3}}{p_{2} A_{2}} \frac{Q^{\prime}}{Q}-\varphi \frac{B_{1}-B_{3}^{\prime}}{p_{2} A_{2}}+\frac{1}{n} \frac{Q^{\prime}}{Q} b-\frac{1}{n} \frac{\left(p_{2} A_{2}\right)^{\prime}}{p_{2} A_{2}} b \\
+\frac{2}{3 n^{2}} \frac{B_{2}+B_{3}}{p_{2} A_{2}} b+\left(\frac{Q^{\prime}}{Q}-\frac{\left(p_{2} A_{2}\right)^{\prime}}{p_{2} A_{2}}+\frac{B_{3}}{p_{2} A_{2}}\right)\left(\varphi^{2}+\varphi^{\prime}+\frac{1}{3 n^{2}} b\right) \\
-\left(\varphi^{3}+3 \varphi \varphi^{\prime}+\varphi^{\prime \prime}+\frac{9 n+4}{3 n^{2}} \varphi b+\frac{3 n+4}{3 n^{2}} b^{\prime}\right) \equiv 0 .
\end{gathered}
$$

By $\mu_{3}(z) \equiv 0$, we have

$$
\begin{aligned}
& 3 n \frac{Q^{\prime}}{Q} \varphi-\left(3 n \frac{\left(p_{2} A_{2}\right)^{\prime}}{p_{2} A_{2}}-\frac{2 B_{2}}{p_{2} A_{2}}-\frac{2 B_{3}}{p_{2} A_{2}}\right) \varphi-\left(\frac{B_{2}}{p_{2} A_{2}} \frac{R^{\prime}}{R}+\frac{2 B_{1}}{p_{2} A_{2}}-\frac{B_{2}^{\prime}}{p_{2} A_{2}}\right) \\
& -b-(3 n+2)\left(\varphi^{2}+\varphi^{\prime}+\frac{1}{3 n^{2}} b\right)-3 n \varphi^{2} \equiv 0 .
\end{aligned}
$$

Noticing the expressions of $\varphi, \frac{B_{1}}{p_{2} A_{2}}, \frac{B_{2}}{p_{2} A_{2}}, \frac{B_{3}}{p_{2} A_{2}}, \frac{B_{1}^{\prime}}{p_{2} A_{2}}, \frac{B_{2}^{\prime}}{p_{2} A_{2}}, \frac{B_{3}^{\prime}}{p_{2} A_{2}}$, we know that the highest powers of $z$ in the left hand side of (28), (29), (30) may, respectively, appear in

$$
\begin{aligned}
& \frac{3 n-1}{27 n^{4}}\left(\alpha_{1}^{\prime}+\alpha_{2}^{\prime}\right)^{2}-\frac{1}{3 n^{2}} \alpha_{1}^{\prime} \alpha_{2}^{\prime}-\frac{3 n+1}{9 n^{4}} b, \\
& \frac{3 n-1}{27 n^{3}}\left(\alpha_{1}^{\prime}+\alpha_{2}^{\prime}\right)^{3}-\frac{1}{3 n} \alpha_{1}^{\prime} \alpha_{2}^{\prime}\left(\alpha_{1}^{\prime}+\alpha_{2}^{\prime}\right)+\frac{6 n^{2}-4}{9 n^{3}}\left(\alpha_{1}^{\prime}+\alpha_{2}^{\prime}\right) b, \\
& \frac{6 n^{2}-2}{9 n^{2}}\left(\alpha_{1}^{\prime}+\alpha_{2}^{\prime}\right)^{2}-2 \alpha_{1}^{\prime} \alpha_{2}^{\prime}-\frac{3 n^{2}+3 n+2}{3 n^{2}} b .
\end{aligned}
$$

Let $\alpha_{1}(z)=a_{p} z^{p}+a_{p-1} z^{p-1}+\cdots+a_{0}, \alpha_{2}(z)=b_{q} z^{q}+b_{q-1} z^{q-1}+\cdots+b_{0}$, where $a_{i}(i=0,1, \ldots, p)$, $b_{j}(i=0,1, \ldots, q)$ are complex numbers, $a_{p} b_{q} \neq 0$ and $p \geq 1, q \geq 1$.

If $p \neq q$, without loss of generality, we assume that $p>q$. Obviously, $\operatorname{deg}_{\infty} b \leq 2 p-2$, otherwise we can get a contradiction from (31) immediately. If $\operatorname{deg}_{\infty} b<2 p-2$, then from (31), we have $\frac{3 n-1}{27 n^{4}}=0$. So $n=\frac{1}{3}$, this is impossible. If $\operatorname{deg}_{\infty} b=2 p-2$, then from (31) and 
(33), we obtain

$$
\frac{\frac{3 n-1}{27 n^{4}}}{\frac{6 n^{2}-2}{9 n^{2}}}=\frac{\frac{3 n+1}{9 n^{4}}}{\frac{3 n^{2}+3 n+2}{3 n^{2}}},
$$

by solving above equation, we have $n= \pm 1$, this is impossible. Next we suppose that $p=q$.

If $\operatorname{deg}_{\infty} b>2 p-2$, then by $\mu_{1}(z) \equiv 0$ and (31), we have $-\frac{3 n+1}{9 n^{4}}=0$, so $n=-\frac{1}{3}$, this is impossible. Thus we may assume that $\operatorname{deg}_{\infty} b \leq 2 p-2$.

If $a_{p}+b_{p}=0$ and $\operatorname{deg}_{\infty} b<2 p-2$, then by $\mu_{1}(z) \equiv 0$ and (31), we have $-\frac{1}{3 n^{2}} p^{2} a_{p} b_{p}=0$, this is impossible. If $a_{p}+b_{p}=0$ and $\operatorname{deg}_{\infty} b=2 p-2$, then by $\mu_{1}(z) \equiv 0, \mu_{3}(z) \equiv 0$ and (31), (33) we have $\frac{2(3 n+1)}{9 n^{4}}=\frac{3 n^{2}+3 n+2}{9 n^{4}}$, hence $n=1$, this is also impossible.

If $a_{p}+b_{p} \neq 0$ and $\operatorname{deg}_{\infty} b<2 p-2$, then by $\mu_{1}(z) \equiv 0, \mu_{3}(z) \equiv 0$ and (31), (33) we have $\frac{2(3 n-1)}{27 n^{4}}=\frac{6 n^{2}-2}{27 n^{4}}$, hence $n=1$, this is a contradiction with the assumption $n \geq 2$. If $a_{p}+b_{p} \neq 0$ and $\operatorname{deg}_{\infty} b=2 p-2$, then from (31), (32) and the assumption $\mu_{1}(z) \equiv 0, \mu_{2}(z) \equiv 0$, we have $-\frac{3 n+1}{9 n^{4}}=\frac{6 n^{2}-4}{9 n^{4}}$, thus $n=\frac{1}{2}$ or $n=-1$, this is impossible. Therefore, $\mu_{1}(z) \equiv 0, \mu_{2}(z) \equiv 0$, $\mu_{3}(z) \equiv 0$ cannot hold simultaneously.

If $\mu_{3}(z) \not \equiv 0$. Then equation (27) can be rewritten as

$$
\mu_{3}(z)\left(f^{\prime}\right)^{2}=-\mu_{1}(z) f^{2}-\mu_{2}(z) f f^{\prime}
$$

Suppose that $f(z)$ has infinitely many zeros, then we know that the multiplicity of each zero of $f(z)$ is not less than 2 , possibly except finitely many zeros of $f(z)$. Let $z_{0}$ be a zero of $f$ with multiplicity $k$, but that is not the zero and pole of $\mu_{1}(z), \mu_{2}(z), \mu_{3}(z)$, then $z_{0}$ is a zero with multiplicity $2 k-2$ in the left side and a zero with multiplicity at least $2 k-1$ in the right side of equation (34). This contradiction lead to that $f$ has at most finitely many zeros.

If $\mu_{3}(z) \equiv 0$, then $\mu_{1}(z) \not \equiv 0$ and $\mu_{2}(z) \not \equiv 0$, equation (27) is simplified to the following form:

$$
\mu_{1}(z) f=-\mu_{2}(z) f^{\prime}
$$

by a similar discussion to above, we see that $f$ has at most finitely many zeros. Thus, $f(z)=$ $q(z) e^{P(z)}$, where $q(z)$ is a rational function and $P(z)$ is a polynomial.

Substituting $f(z)=q(z) e^{P(z)}$ into the equation (3) yields

$$
q(z)^{n}\left(q^{\prime}(z)+q(z) p^{\prime}(z)\right) e^{(n+1) P(z)}+R(z)=p_{1}(z) e^{\alpha_{1}(z)}+p_{2}(z) e^{\alpha_{2}(z)}
$$

If $\alpha_{1}^{\prime}(z) \not \equiv \alpha_{2}^{\prime}(z)$, it follows from Lemma 1 that $R(z) \equiv 0$. Furthermore, either $(n+1) P(z)=$ $\alpha_{1}(z)+C_{1}, q(z)^{n}\left(q^{\prime}(z)+q(z) P^{\prime}(z)\right)=D_{1} p_{1}(z), p_{2}(z) \equiv 0$ or $(n+1) P(z)=\alpha_{2}(z)+C_{2}, q(z)^{n}\left(q^{\prime}(z)+\right.$ $\left.q(z) P^{\prime}(z)\right)=D_{2} p_{2}(z), p_{1}(z) \equiv 0$, where $C_{1}, C_{2}, D_{1}, D_{2}$ are constants and $D_{1} e^{C_{1}}=D_{2} e^{C_{2}}=$ 1. This is a contradiction with the assumption $p_{1}, p_{2}$ are nonzero rational functions. If $\alpha_{1}^{\prime}(z) \equiv \alpha_{2}^{\prime}(z)$, then $\alpha_{2}(z)=\alpha_{1}(z)+C$, where $C$ is a constant, and it follows from Lemma 1 that $R(z) \equiv 0$ and the equation (3) is reduced to the following form:

$$
f^{n} f^{\prime}=\left(p_{1}+p_{2} e^{C}\right) e^{\alpha_{1}(z)}
$$


By Theorem A, we have

$$
f(z)=q(z) e^{\frac{\alpha_{1}(z)}{n+1}}
$$

where $q(z)$ is a rational function with $q^{n}\left((n+1) q^{\prime}+q \alpha_{1}^{\prime}\right)=(n+1)\left(p_{1}+p_{2} e^{\alpha_{2}(z)-\alpha_{1}(z)}\right)$.

\section{Competing interests}

The author declares that there is no conflict of interests regarding the publication of the article.

\section{Acknowledgements}

The author would like to thank the anonymous referees for their valuable comments and suggestions. The research was supported by Natural Science Foundation of Jiangsu Province (BK20140767), Natural Science Foundation of the Jiangsu Higher Education Institutions (14KJB1 10004) and Qing Lan Project of Jiangsu Province.

Received: 19 August 2016 Accepted: 17 November 2016 Published online: 24 November 2016

References

1. Hayman, WK: Meromorphic Functions. Clarendon Press, Oxford (1964)

2. Laine, I: Nevanlinna Theory and Complex Differential Equations. Walter de Gruyter, Berlin (1993)

3. Li, P, Yang, CC: On the nonexistence of entire solutions of certain type of nonlinear differential equations. J. Math. Anal. Appl. 320, 827-835 (2006)

4. Li, P: Entire solutions of certain type of differential equations II. J. Math. Anal. Appl. 375, 310-319 (2011)

5. Liao, LW, Yang, CC, Zhang, JJ: On meromorphic solutions of certain type of non-linear differential equations. Ann. Acad. Sci. Fenn., Math. 38, 581-593 (2013)

6. Liao, LW, Ye, Z: On solutions to nonhomogeneous algebraic differential equations and their application. J. Aust. Math. Soc. 97, 391-403 (2014)

7. Yang, CC, Yi, HX: Uniqueness Theory of Meromorphic Functions. Kluwer Academic, Dordrecht (2003)

8. Yang, CC, Ye, Z: Estimates of the proximate function of differential polynomials. Proc. Jpn. Acad., Ser. A, Math. Sci. 83, 50-55 (2007)

\section{Submit your manuscript to a SpringerOpen ${ }^{\ominus}$ journal and benefit from:}

- Convenient online submission

Rigorous peer review

- Immediate publication on acceptance

- Open access: articles freely available online

- High visibility within the field

- Retaining the copyright to your article 